\title{
Práticas integrativas e complementares no SUS: os benefícios do Yoga e da Meditação para a saúde do corpo e da alma
}

\author{
Alexsandro Melo Medeiros ${ }^{1}$
}

\section{RESUMO}

Este artigo propõe uma reflexão sobre as práticas integrativas e complementares à partir da análise de técnicas como o yoga e a meditação. A escolha de ambas justifica-se: pela impossibilidade de analisar todas as práticas reconhecidas pela Política Nacional das Práticas Integrativas e Complementares no SUS no espaço de um artigo; a ampla quantidade de estudos já existentes reconhecendo sua eficácia terapêutica; e sua fundamentação, religiosa e espiritual, que nos permite discutir as complexas relações entre o binômio religiosidade/saúde. Como procedimento metodológico adota-se a pesquisa bibliográfica tendo como referencial teórico autores como: Benson (2000), Feuerstein (2006), Goleman (1997), Rodrigues, et. al., (2006), Santos (2010), Siegel (2010). Conclui-se que o yoga e a meditação podem oferecer aos pacientes do SUS tanto uma vivência com o sagrado quanto possuem eficácia terapêutica comprovada para a saúde do corpo e a saúde da alma.

Palavras-chave: Yoga, Meditação, Religiosidade, Saúde.

\section{INTEGRATIVE AND COMPLEMENTARY PRACTICES IN SUS: THE BENEFITS OF YOGA AND MEDITATION FOR THE HEALTH OF BODY AND SOUL}

\begin{abstract}
This article proposes a reflection on integrative and complementary practices from the analysis of techniques such as yoga and meditation. The choice of both is justified by the impossibility of analyzing all the

Mestre em Filosofia pela Universidade Federal de Pernambuco. Doutorando do Programa de Pós-Graduação Sociedade e Cultura na Amazônia. Professor de Filosofia da Universidade Federal do Amazonas. Bolsista e Pesquisador da Fundação de Amparo à Pesquisa do Estado do Amazonas. E-mail: alexsandromedeiros@ufam.edu.br. Lattes: lattes.cnpq. br/6947356140810110.
\end{abstract}


practices recognized by the National Policy of Integrative and Complementary Practices in the SUS in the space of an article; the vast amount of studies already in existence recognizing its therapeutic efficacy; and its religious and spiritual foundation, which allows us to discuss the complex relationships between the binomial religiosity/health. As a methodological procedure, the bibliographical research is based on the following authors: Benson (2000), Feuerstein (2006), Goleman (1997), Rodrigues, et. al., (2006), Santos (2010), Siegel (2010). It is concluded that yoga and meditation can offer to the patients of the SUS an experience with the sacred and have proven therapeutic efficacy for the health of the body and the soul.

Keywords: Yoga, Meditation, Religiosity, Health.

\section{Introdução}

Em 2006 o Ministério da Saúde aprovou, através da portaria 971 de 03 de maio, a Política Nacional de Práticas Integrativas e Complementares (PNPIC). Inicialmente a PNPIC previa a utilização de algumas poucas técnicas como: a Medicina Tradicional Chinesa-Acupuntura ${ }^{2}$, Homeopatia $^{3}$, o uso de Plantas Medicinais e Fitoterapia ${ }^{4}$ e o Termalismo Social/

2 "A Medicina Tradicional Chinesa caracteriza-se por um sistema médico integral, originado há milhares de anos na China. Utiliza linguagem que retrata simbolicamente as leis da natureza e que valoriza a inter-relação harmônica entre as partes visando à integridade. Como fundamento, aponta a teoria do Yin-Yang, divisão do mundo em duas forças ou princípios fundamentais, interpretando todos os fenômenos em opostos complementares [...] A acupuntura é uma tecnologia de intervenção em saúde que aborda de modo integral e dinâmico o processo saúde-doença no ser humano, podendo ser usada isolada ou de forma integrada com outros recursos terapêuticos. Originária da medicina tradicional chinesa (MTC), a acupuntura compreende um conjunto de procedimentos que permitem o estímulo preciso de locais anatômicos definidos por meio da inserção de agulhas filiformes metálicas para promoção, manutenção e recuperação da saúde, bem como para prevenção de agravos e doenças" (MINISTÉRIO DA SAÚDE, 2006).

3 "A homeopatia, sistema médico complexo de caráter holístico, baseada no princípio vitalista e no uso da lei dos semelhantes foi enunciada por Hipócrates no século IV a.C. Foi desenvolvida por Samuel Hahnemann no século XVIII. Após estudos e reflexões baseados na observação clínica e em experimentos realizados na época, Hahnemann sistematizou os princípios filosóficos e doutrinários da homeopatia em suas obras Organon da Arte de Curar e Doenças Crônicas” (MINISTÉRIO DA SAÚDE, 2006).

4 "A fitoterapia é uma "terapêutica caracterizada pelo uso de plantas medicinais em suas diferentes formas farmacêuticas, sem a utilização de substâncias ativas isoladas, ainda que de origem vegetal". O uso de plantas medicinais na arte de curar é uma forma de tratamento de origens muito antigas, relacionada aos primórdios da medicina e fundamentada no acúmulo de informações por sucessivas gerações. Ao longo dos séculos, produtos de origem vegetal constituíram as bases para tratamento de diferentes doenças" (MINISTÉRIO DA SAÚDE, 2006). 
Crenoterapia $^{5}$. Em 2017 ocorre um significativo incremento das técnicas abordadas através das portarias: 145, de 11 de janeiro e 849 de março, ambas de 2017. As duas portarias incluem na PNPIC novas técnicas dentre as quais podemos destacar: $\operatorname{arteterapia}^{6}$, meditação $^{7}$, musicoterapia $^{8}$, reiki $^{9}$, medicina ayurveda ${ }^{10}$, shantala $^{11}$ e o yoga ${ }^{12}$.

5 "O uso das Águas Minerais para tratamento de saúde é um procedimento dos mais antigos, utilizado desde a época do Império Grego. Foi descrita por Heródoto (450 a.C.), autor da primeira publicação científica termal. O termalismo compreende as diferentes maneiras de utilização da água mineral e sua aplicação em tratamentos de saúde. A crenoterapia consiste na indicação e uso de águas minerais com finalidade terapêutica atuando de maneira complementar aos demais tratamentos de saúde" (MINISTÉRIO DA SAÚDE, 2006).

6 "É uma prática que utiliza a arte como base do processo terapêutico. Faz uso de diversas técnicas expressivas como pintura, desenho, sons, música, modelagem, colagem, mímica, tecelagem, expressão corporal, escultura, dentre outras. Pode ser realizada de forma individual ou em grupo. Baseia-se no princípio de que o processo criativo é terapêutico e fomentador da qualidade de vida" (MINISTÉRIO DA SAÚDE, 2017).

7 "É uma prática de harmonização dos estados mentais e da consciência, presente em inúmeras culturas e tradições. Também é entendida como estado de Samadhi, que é a dissolução da identificação com o ego e total aprofundamento dos sentidos, o estado de "êxtase"” (MINISTÉRIO DA SAÚDE, 2017).

8 "É a utilização da música e seus elementos (som, ritmo, melodia e harmonia), em grupo ou de forma individualizada, num processo para facilitar e promover a comunicação, relação, aprendizagem, mobilização, expressão, organização e outros objetivos terapêuticos relevantes, no sentido de alcançar necessidades físicas, emocionais, mentais, sociais e cognitivas. A Musicoterapia objetiva desenvolver potenciais e restabelecer funções do indivíduo para que possa alcançar uma melhor integração intra e interpessoal e, consequentemente, uma melhor qualidade de vida" (MINISTÉRIO DA SAÚDE, 2017).

9 "É uma prática de imposição de mãos que usa a aproximação ou o toque sobre o corpo da pessoa com a finalidade de estimular os mecanismos naturais de recuperação da saúde. Baseado na concepção vitalista de saúde e doença também presente em outros sistemas terapêuticos, considera a existência de uma energia universal canalizada que atua sobre o equilíbrio da energia vital com o propósito de harmonizar as condições gerais do corpo e da mente de forma integral" (MINISTÉRIO DA SAÚDE, 2017)

10 "É considerado uma das mais antigas abordagens de cuidado do mundo, foi desenvolvido na Índia durante o período de 2000-1000 a.C. Utilizou-se de observação, experiência e os recursos naturais para desenvolver um sistema único de cuidado. Ayurveda significa a Ciência ou Conhecimento da Vida. Este conhecimento estruturado agrega em si mesmo princípios relativos à saúde do corpo físico, de forma a não desvinculá-los e considerando os campos energético, mental e espiritual” (MINISTÉRIO DA SAÚDE, 2017).

11 "É uma prática de massagem para bebês e crianças, composta por uma série de movimentos pelo corpo, que permite o despertar e a ampliação do vínculo cuidador e bebê. Além disso, promove a saúde integral, reforçando vínculos afetivos, a cooperação, confiança, criatividade, segurança, equilíbrio físico e emocional. Promove e fortalece o vínculo afetivo, harmoniza e equilibra os sistemas imunológico, respiratório, digestivo, circulatório e linfático. Permite ao bebê e à criança a estimulação das articulações e da musculatura auxiliando significativamente no desenvolvimento motor, facilitando movimentos como rolar, sentar, engatinhar e andar" (MINISTÉRIO DA SAÚDE, 2017).

12 "É uma prática que combina posturas físicas, técnicas de respiração, meditação e relaxamento. Atua como uma prática física, respiratória e mental. Fortalece o sistema músculo-esquelético, estimula o sistema endócrino, expande a capacidade respiratória e exercita o sistema cognitivo. Um conjunto de ásanas (posturas corporais) pode reduzir a 
A Política Nacional de Práticas Integrativas e Complementares vai na contramão do modelo biomédico hegemônico oficial, que tende a ignorar práticas alternativas e tradicionais de curas, fundamenta-se em um reducionismo biológico, na cisão mente-corpo, desqualifica o conhecimento popular, tradicional e religioso sobre as doenças e seu tratamento. Mesmo que a biomedicina traga contribuições significativas para as condições da saúde, aumentando a expectativa de vida e evitando epidemias, ela amputa o olhar sobre o doente e sua subjetividade, o que traz uma série de implicações para uma compreensão mais integral do ser humano, como propõe as práticas alternativas e complementares, que levam em consideração fatores biopsicossociais e até mesmo espirituais, como se pretende demonstrar neste artigo.

Considerando, portanto, o reconhecimento de tais práticas por parte do Ministério da Saúde, julga-se relevante um melhor conhecimento e entendimento sobre tais técnicas. Todavia, diante da impossibilidade de abordar cada uma destas práticas no âmbito de um artigo, optou-se pela análise do Yoga e da Meditação por duas razões: a primeira, em função da ampla quantidade de estudos já existentes reconhecendo sua eficácia terapêutica, como será possível perceber no vasto referencial teórico apresentado no artigo; a segunda, considerando que a prática do Yoga e da Meditação possuem em sua essência uma base religiosa e espiritual, sua análise nos permite discutir o binômio religiosidade/ saúde. Pretende-se, portanto, enfatizar as complexas relações entre o fenômeno religioso e reconhecer a sua importância e os benefícios que tais práticas desempenham na área da saúde.

Ao abordar a prática do Yoga e da Meditação toma-se como direcionamento a importância de considerar a questão espiritual sob uma prisma voltado para a implementação de ações no campo da saúde que considere o ser humano de forma integral superando, assim, o modelo biomédico da medicina oficial.

As práticas integrativas e complementares, à medida que se relacionam com o fenômeno religioso, têm muito a contribuir com os estudos na área das ciências das religiões, sobretudo no campo das expressões

dor lombar e melhorar Para harmonizar a respiração, são praticados exercícios de controle respiratório denominados de prânâyâmas. Também, preconiza o autocuidado, uma alimentação saudável e a prática de uma ética que promova a não-violência" (MINISTÉRIO DA SAÚDE, 2017). 
religiosas orientais e espiritualistas, considerando que "investigar quem produz e participa desse conhecimento [...] o funcionamento do corpo-mente humano, também deve fazer parte das ciências que compõem os estudos religiosos" (SIMÕES, 2011, p. 11).

Ao procurar descrever a experiência religiosa como um fenômeno presente nos processos de tratamento, cura e prevenção da saúde, através de técnicas como o Yoga, a Meditação e tantas outras, as práticas integrativas e complementares se inserem no campo da fenomenologia religiosa, exigindo a formulação de descrições criteriosas e buscando o seu significado.

Como metodologia utilizou-se a pesquisa bibliográfica, a partir de um amplo referencial teórico sobre a temática escolhida da qual é importante ressaltar que as pesquisas apresentadas foram, algumas, resultado de pesquisas em nível de mestrado e doutorado e outras, publicadas em periódicos reconhecidos nacional e internacionalmente, como: International Journal of Psychology and Behavioral Science, Journal of Personality and Social Psychology, Psychosomatic Medicine, Revista Brasileira de Psiquiatria, Boletim da Academia Paulista de Psicologia, Revista Brasileira de Terapias Cognitivas, Sociedad y Religión; e portais como: PubMed e Scielo.

A escolha dos autores utilizados se deu em decorrência da pesquisa nos portais PubMed e Scielo. A partir dessa pesquisa selecionou-se os artigos que de alguma forma relacionavam a prática da meditação e do yoga com situações de saúde/doença, aliado ao referencial teórico de autores já conhecidos pelos seus estudos na área como: Benson (2000), Feuerstein (2006), Goleman (1997), Rodrigues, et. al., (2006), Santos (2010), Siegel (2010).

Com base neste referencial este artigo propõe uma análise dos benefícios da prática do yoga (primeira parte do artigo) e da meditação (segunda parte do artigo) para a saúde do corpo e da alma. E conclui que o yoga e a meditação podem oferecer aos pacientes do SUS tanto uma vivência com o sagrado quanto possuem eficácia terapêutica comprovada para a saúde do corpo e a saúde da alma. 


\section{Os Benefícios do Yoga para a Saúde do Corpo e da Alma}

O Yoga pode ser definido como uma filosofia oriental associada não apenas a uma prática física ou de posturas corporais e exercícios respiratórios ${ }^{13}$, mas seu objetivo é a busca de uma vivência com o sagrado que conduz a união entre o Corpo-Mente-Alma e entre a alma e Deus. O yoga "além de ser uma filosofia ou uma visão de mundo, é uma prática mente-corpo, que abrange técnicas fisiológicas e psíquicas, e visa chegar ao estado de identificação do ser com a sua essência, através da ausência de flutuações mentais" (SIEGEL, 2010, p. 28). A palavra Yoga é de origem sânscrita e pode ser traduzida por união, no sentido de integração (LIMA, 2010, p. 102). Etimologicamente a palavra yoga deriva da raiz sânscrita $y u j$ que quer dizer "conjugar, juntar, jungir" (FEURSTEIN, 2006, p. 39).

Yoga é o caminho e o caminhar que conduz a Deus. Yoga ensina como as pessoas podem viver para Deus com Deus e em Deus [...] O Bhagvada (sic) Gita fala que "Aquele que se tenha desapegado dos contatos externos encontra a felicidade em Atman (Alma); tendo alcançado a união (Yoga) com Brahman (Deus) desfruta a bem-aventurança eterna" (TEOTIA; TEOTIA, 2000, p. 15-16 - grifos no original).

A origem das teorias e práticas do Yoga são desconhecidas, mas certamente o legado que nos foi transmitido pelos textos sânscritos remontam a mais de mil anos antes da era cristã, desde o chamado período védico, bramânico e pós védico. Neste último é onde surgem os primeiros Upanishads e Brâhmanas. Desde então o conhecimento e a prática do Yoga é transmitido ao longo das eras como no período clássico, "formando-se as seis grandes escolas clássicas de filosofia: 1. Yoga-Sutras de Patânjali; 2. Brahma-Sutra; 3. Badarayana; 4. Samkhya-Karika; 5. Ishvara-Krishna e 6. Vaishnavismo" (LIMA, 2010, p. 27).

13 Não existe apenas uma corrente do Yoga ou caminho para a realização do indivíduo. Há várias correntes e a corrente mais conhecida, direcionada para o domínio e controle do corpo físico através das posturas (asanas) é o Hatha Yoga. Mas além desta existem ainda o Yoga do conhecimento (Jnana), o Yoga da devoção (Bhakti), o Yoga do serviço (Karma) e ainda o "Raja Yoga (integral, pois combina os quatro tipos precedentes)" (PEÇANHA; CAMPANA, 2010, p. 203). "Atualmente, o Hatha Yoga é o ramo mais praticado no mundo por pessoas que o procuram pelos benefícios físicos, mas desconhecem seu objetivo tradicional: a autorrealização" (LIMA, 2010, p. 37). 
Nos últimos anos a prática do Yoga tem sido adotada por milhões de pessoas, em um processo de transculturalização ou, hibridismo cultural - para usar a expressão de Hall (2006) -, marcado pela inclusão de elementos filosóficos, éticos e religiosos. Por isso essa prática não pode passar despercebida pelos estudos na área das ciências das religiões que, enquanto ciência, tem o dever de se debruçar sobre o fenômeno religioso em suas múltiplas e variadas expressões, neste caso da tradição oriental e, de modo mais específico, como essa prática associa espiritualidade e saúde ${ }^{14}$.

Aplicada ao campo da saúde, a yogaterapia foi um termo cunhado desde o início do século XX, mais precisamente na década de 1920, por Swami Kuvalayananda (ALTER, 2004, p. 73-108), para dar ênfase à prática do yoga como um recurso terapêutico para determinados sintomas, problemas orgânicos, físicos e patológicos. Um tipo de terapia que se espalhou internacionalmente "[...] com o surgimento de centros de yogaterapia, inclusão da prática de yoga e meditação em programas de câncer de hospitais, centros de medicina alternativa afiliados e o estabelecimento de uma nova geração de clínicos chamados yogaterapeutas" (VORKAPIC; RANGÉ, 2011, p. 51).

Posteriormente, Mircea Eliade, historiador da religião, defendeu a primeira tese de doutorado sobre yoga no ocidente, em Bucareste, em 1933, publicada como livro, intitulado 'Yoga, Imortalidade e Liberdade'. Sua obra consta de oito capítulos, sendo que, no primeiro, parte da dor da existência, pergunta se a liberação é possível e descreve, nos capítulos subsequentes, as técnicas, a filosofia, a simbologia e o yoga relacionado ao bramanismo, hinduísmo, budismo, tantrismo, à alquimia e aos aborígenes indianos (SIEGEL, 2010, p. 27).

Siegel (2010, p. 91-103) é a autora de uma importante pesquisa sobre Yoga no Brasil de estudos acadêmicos publicados no banco de teses e dissertações da CAPES no período 2004-2008. A pesquisadora constatou a existência de vários estudos em diferentes áreas desde a

14 Sobre a associação da temática religiosa com a saúde veja, por exemplo, a proposta de incluir a temática "Fisiologia e Saúde" na área de estudos das ciências da religião ou como o advento da neurociência tem possibilitado construir uma base empírica para os estudos das ciências de religião, como ressalta Simões (2011). 
Antropologia Social e a Educação, até a Educação Física, Enfermagem e Nutrição.

Outros estudos associam a prática do yoga a vários benefícios para a saúde do corpo físico e também para aspectos relacionados de ordem psíquica (RODRIGUES, et. al., 2006) tais como: redução do estresse (GHAROTE, 2000; PASHARD, 2004) e da ansiedade (JAVNBAKHT, M.; HEJAZI, K.; GHASEMI, M., 2009; VORKAPIC; RANGÉ, 2011), fortalecimento do sistema imunológico, melhora do sono, do funcionamento do sistema respiratório e comportamento cardiovascular (LEITE, 1999). Simões (2011, p. 15) ressalta ainda "que as atividades corporais religiosas têm sido objeto de pesquisa em laboratórios e centros acadêmicos de diversas universidades no mundo".

No Brasil merece destaque os trabalhos da pesquisadora Elisa Kozasa, do Instituto Israelita de Ensino e Pesquisa Albert Einstein em São Paulo, cujas pesquisas têm contribuído para a abertura da comunidade científica sobre práticas como o Yoga, a Meditação, o Tai Chi, abordando principalmente mudanças neurofisiológicas e alterações no nível de cortisol (KOZASA, 2006; KOZASA, et. al., 2013; KOZASA, et. al., 2012; KOZASA, et. al., 2011; KOZASA, et. al., 2010).

Lima (2010, p. 13) relata um caso pessoal em que através da prática do Yoga conseguiu o restabelecimento da saúde de um filho diagnosticado como portador de asma desde o primeiro mês de vida. “Ao usar os Pranayamas ou os exercícios para o desenvolvimento de sua respiração, juntamente com as modificações alimentares, consegui de imediato, que ele saísse da crise asmática"15.

Como técnica terapêutica o Yoga pode ser praticado por qualquer pessoa, de qualquer religião, inclusive aquelas que não tenham religião ou se declarem ateus. Todavia, quando nos aprofundamos em sua filosofia, não é difícil perceber que o Yoga é muito mais do que uma prática física de posturas corporais e exercícios respiratórios. Seu objetivo é promover no seu praticante uma vivência que conduz a união entre o Corpo, a Mente, a Alma e Deus, e visa conduzir a alma ao estado de identificação com a sua essência.

15 Para mais detalhes sobre pesquisas relacionando a prática do Yoga com o tratamento da asma ver: JAIN, et. al., 1991; MILLER, 2001, SINGH, et. al., 1990. 
O objetivo dos ensinamentos yoguicos, por exemplo, em realizar posturas físicas combinadas com exercícios respiratórios e meditação, não é para melhorar a saúde ou produzir experiências místicas aos seus praticantes apenas. O seu intuito está em conduzir o devoto ou o praticante de Yoga (sadhaka/neófito) à kaivalya (lit.libertação), libertando-o das suas aflições ou do seu sofrimento. As experiências fenomênicas e a saúde que advém destas práticas são consequências e não o fim em si-mesmas (SIMÕES, 2011, p. 16).

No âmbito do religioso e do sagrado, o Yoga é muito mais do que uma prática voltada para o desenvolvimento da flexibilidade do corpo, do equilíbrio da saúde física e da concentração mental. O Yoga possui um grande valor terapêutico/medicinal que não pode ser desvinculado de seu aspecto espiritual. O Yoga envolve todos estes fatores: controle do corpo, da atenção, da vida, exercícios respiratórios, posturas psicofísicas, preceitos éticos, sistema de crenças, experiências sensoriais, transcendência do eu, estado contemplativo, comunhão com o sagrado, união com Deus. Na verdade, como ressalta Simões (2011, p. 21), ao analisar a história e doutrina do Yoga: "torna-se bastante improvável considerá-lo sem o seu caráter religioso, como se vê explicitamente na sua literatura".

\section{Os Benefícios da Meditação para a Saúde do Corpo e da Alma}

A meditação é uma prática bastante antiga, cujos "métodos e objetivos variam, mas, geralmente, nos contextos tradicionais, a meditação representa uma abertura para a comunhão com forças divinas, por meio de autoapaziguamento e neutralização dos pensamentos incessantes" (MORAES, 2015, p. 1619). Sua origem remonta às tradições orientais, estando especialmente relacionada à prática do yoga, do hinduísmo, do budismo e suas derivações como sinônimo de busca espiritual. Como destaca Lemos (2014, p. 47), na meditação, sobretudo do ponto de vista do pensamento oriental: "há valorização da intuição, observação do mundo interior, busca de sabedoria, foco em ser, religiosidade e misticismo. A prática geralmente é vinculada a um contexto religioso e à busca espiritual" 
A meditação consiste em um conjunto de técnicas que treinam a focalização da atenção e da mente de tal modo que seja capaz de produzir maior integração entre mente, corpo, o mundo externo e capaz de produzir efeitos psicossomáticos. Todavia, não existe uma única vertente da meditação. Na tradição budista tem-se a meditação Vipassana e a Anapana: a primeira é uma prática de observação do momento presente, enquanto a segunda tem o foco na respiração (ADHIKARI, 2012). Foi a partir de uma adaptação da meditação anapana que Jon Kabat-Zinn, biólogo e professor da Universidade de Medicina de Massachussets,

integrou seus conhecimentos budistas à ciência médica ocidental e criou, assim, a prática denominada mindfulness, que em português foi traduzida como prática de atenção plena. Kabat-Zinn criou o programa de Redução do Estresse baseado na Atenção Plena. Esta prática é largamente utilizada em pesquisas científicas e difundida nas instituições de saúde dos Estados Unidos (LEMOS, 2014, p. 48).

$\mathrm{Na}$ década de setenta a meditação começou a ser investigada cientificamente no Ocidente sendo que as pesquisas e publicações são predominantemente estrangeiras. Mas a partir desse acervo teórico e experimental vários pesquisadores brasileiros têm se debruçado sobre o fenômeno da meditação, inclusive considerando a possibilidade de aplicação desta técnica de forma terapêutica para melhoria e prevenção da saúde.

Merece destaque a este respeito a pesquisa de Lemos (2014), realizada no Instituto Nacional de Traumatologia e Ortopedia (INTO) do Rio de Janeiro, que utilizou a prática da meditação de atenção plena ${ }^{16}$, meditação da consciência amorosa ${ }^{17}$ e o método Feldenkrais ${ }^{18}$, com o objetivo de avaliar a relação entre a prática da meditação e as repercussões na saúde e no bem estar individual de seus praticantes.

16 "utilizando o protocolo de Kabat-Zinn, biólogo que adaptou meditações da tradição budista para utilização na saúde, sem vínculo religioso" (LEMOS, 2014, p. 16).

17 "adaptada a partir da meditação budista-Metta Bhavana, por Vidyamala Burch, fundadora da Breathworks" (LEMOS, 2014, p. 15).

18 "método este desenvolvido por Moshé Feldenkrais, russo que praticava artes marciais e desenvolveu esta prática mente-corpo, onde a partir da consciência pelo movimento busca-se estar no presente, percebendo-se e atuando no seu processo" (LEMOS, 2014, p. 15). 
Tivemos como resultados a melhora da dor e a relação dos participantes com seu quadro álgico; melhora do sono; observamos que a meditação associada ao método Feldenkrais ${ }^{\circledR}$ promoveu autocuidado; teve impacto positivo nos relacionamentos dos participantes e modificou a visão de mundo; e favoreceu a apropriação da saúde através da disseminação da prática. É importante destacar a inter-relação dos resultados (LEMOS, 2014, p. 17).

Isso porque, segundo a pesquisadora, a prática da meditação gera redução dos níveis de cortisol e adrenalina, o que favorece a ativação do sistema nervoso parassimpático, reduz a ansiedade e o estresse, possibilitando a melhora do sono e da dor.

Para Lemos (2014) a prática da meditação de atenção plena não apenas tem efeitos sobre a atenção como o próprio nome sugere, seja desenvolvendo a qualidade da atenção ou a autorregulação da atenção, mas também vem se mostrando eficaz para melhoria da qualidade da vida na medida em que ajuda na redução da dor, do estresse, melhora o quadro depressivo e auxilia no desenvolvimento de uma relação mais amorosa consigo mesmo e com os outros

A prática da meditação tem sido estudada em associação a vários fatores, seja na melhora da qualidade de vida, do humor, da saúde ou do bem-estar psicológico (CARDOSO, et. al., 2004; CASEY; BENSON, 2004; GOLEMAN, 1997).

Os efeitos da meditação começaram a ser estudados de forma científica através das pesquisas do doutor Herbert Benson, professor e cardiologista da Faculdade de Medicina da Universidade de Harvard nos E.U.A. (SANTOS, 2010). Benson é autor do livro The Relaxation Response (BENSON; KLIPPER, 2000) onde aborda os benefícios da meditação no tratamento e recuperação de seus pacientes e "[...] sistematiza os resultados de quase uma década de suas pesquisas iniciadas na década de 1960 sobre a possibilidade de práticas meditativas controlarem processos químicos-fisiológicos do corpo" (TONIOL, 2015, p. 119).

Uma outra figura de destaque no campo da pesquisa dos efeitos da meditação na área da saúde, especificamente a partir da meditação budista, é o neurocientista americano Richard Davison, da Universidade de Wisconsin-Madison. 
Em 2006, munido com imagens de cérebros de monges tibetanos, Richard Davidson comprovou que a meditação budista altera a estrutura e a densidade do cérebro, além de mudar o padrão das ondas cerebrais. Com número ínfimo de participantes para a dimensão subjetiva do problema, a pesquisa de Davidson conseguiu convencer a comunidade científica de que a meditação faz bem. As imagens de ressonância magnética indicaram o desenvolvimento da região do cérebro responsável pelas emoções "positivas" para aqueles que meditavam há mais tempo (MORAES, 2015, p. 1622).

A possibilidade de avaliar os efeitos da meditação na atividade cerebral hoje é possível graças a instrumentos como o eletroencefalograma, imageamento por ressonância magnética e tomografia computadorizada. Como é o caso da pesquisa de Luders, et. al., (2009) que aponta para evidências de melhorias induzidas pela meditação no bem-estar psicológico e fisiológico e, com o apoio de dados de ressonância magnética de alta resolução de 44 indivíduos (22 meditadores e 22 indivíduos no grupo de controle), detectou, por exemplo, mudanças na macroestrutura cerebral, como o aumento da matéria cinzenta em meditadores no córtex orbito-frontal direito e do hipocampo direito, embora concluam que análises futuras sejam necessárias para estabelecer uma ligação causal entre a prática de meditação e a anatomia do cérebro. Nas palavras dos autores: "os efeitos semelhantes na macroestructura cerebral são prováveis como conseqüência da meditação que 'tradicionalmente requer um compromisso de longo prazo com a prática diária"” (id., ibidem, p. 2 - tradução nossa ${ }^{19}$ ).

A figura 1 abaixo (id., ibidem, p. 14) corresponderia a um exemplo de como o córtex orbito-frontal direito (painel esquerdo), tálamo direito (painel central) e giro temporal inferior esquerdo (painel direito), demonstram que a massa cinzenta é maior em meditadores em comparação com o grupo de controle ${ }^{20}$.

19 "similar effects on cerebral macro-structure are likely as a consequence of meditation which 'traditionally requires a long-term commitment to daily practice"”.

20 "We detected one large cluster of significantly increased GM in meditators compared to controls in the right orbito-frontal cortex (Figure 1, left panel). More specifically, this cluster is located at the border between inferior and middle frontal gyrus (orbital sections) and in approximate distance to Brodmann areas (BA) 11, 12 and 47" (LUDERS, et. al., 2009, p. 676). "Detectamos um grande conjunto de GM [massa cinzenta] significativamente aumentado em meditadores em comparação com controles no córtex direito orbitofrontal (Figura 1, painel esquerdo). Mais especificamente, este cluster está localizado na borda entre o giro frontal inferior e médio (seções orbitais) e em distância aproximada das áreas Brodmann (BA) 11, 12 e 47" (tradução nossa). 


\section{Figura 1 - Larger Gray Matter volumes in meditators ${ }^{21}$}

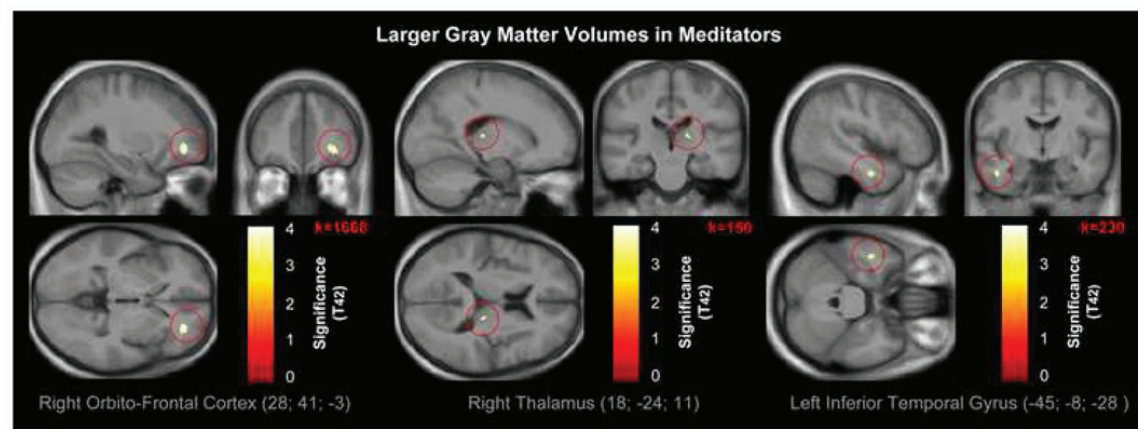

Estudos sobre a prática meditativa associado à diminuição da ansiedade foram realizados por Brown e Ryan (2003), Galvin, et. al., (2006). Davidson, et. al., (2003) ressaltam o efeito da meditação sobre a cognição como: preservação da memória e aumento da capacidade de atenção. No âmbito psicológico e psiquiátrico a meditação tem auxiliado em sintomas como "fobia social" (BOGELS; SIJBERS; VONCKEN, 2006) e na prevenção de comportamento suicida (WILLIAMS; SWALES, 2004).

Sbissa (2010) avaliou o efeito da meditação mindfulness (ou atenção plena) sobre a variabilidade da frequência cardíaca em alunos de um curso técnico em enfermagem,

Entre os achados da presente pesquisa, observou-se que a meditação provocou uma queda na frequência cardíaca (FC), pois o grupo experimental (GE) apresentou um aumento significativo no valor de RR [Intervalo Entre Duas Ondas R], enquanto que o grupo controle (GC) não apresentou o mesmo resultado. Esse resultado indica que o fato de ficar parado/imóvel não foi suficiente para causar o decréscimo na $\mathrm{FC}$, uma vez que o GC não o apresentou (id., ibidem, p. 77).

Sugere o pesquisador que, considerando a meditação como uma técnica cognitiva utilizada para promover um relaxamento psicológico e fisiológico, "a meditação possa ser oferecida como recurso psicológico complementar para redução do estresse em alunos de cursos técnicos" (id., ibidem, p. 21).

${ }^{21}$ Maiores volumes de matéria cinzenta em meditadores (tradução nossa). 
Tal como no Yoga, o objetivo da prática meditativa não é apenas em vista de ter um corpo saudável: "homens e mulheres desempenham tais atos há milênios com a intenção e atenção ao divino (Deus, Ishvara, Brahma, Alá, Oxossi), em busca de respostas das vicissitudes de suas vidas e obtenção do autoconhecimento" (SIMÕES, 2011, p. 16). A percepção da prática meditativa como algo sagrado é tão marcante que Simões (2011, p. ) ressalta como, no período do Yoga Pré-moderno ou Pós-clássico: "os yogues realizados não eram cremados quando morriam [...] mas enterrados em postura de meditação, tornando-se o local sagrado para visitação, pois não se guardava ali um cadáver, mas o corpo de um Liberto em nível de infinita meditação".

\section{Considerações Finais}

As Práticas Integrativas e Complementares constituem hoje um importante aliado no fortalecimento das políticas de saúde, com um olhar diferenciado sobre os sistemas biomédicos hegemônicos. Instituída desde 2006, a Política Nacional de Práticas Integrativas e Complementares amplia-se consideravelmente em 2017 com o reconhecimento de novas práticas dentre as quais inclui-se o yoga e a meditação.

Com a PNPIC o Sistema Único de Saúde brasileiro, ao invés de ignorar práticas alternativas e tradicionais de curas, reconhece sua importância cuja eficácia tem sido comprovada ao longo dos anos através de várias pesquisas científicas transformadas em dissertações de mestrado, teses de doutorado e publicadas em periódicos reconhecidos nacional e internacionalmente.

É o que podemos facilmente constatar quando se considera o Yoga e a Meditação e os benefícios que ambas proporcionam para a saúde do corpo físico e mais além, para a saúde da própria alma, já que ambas as práticas tem como base, fundamentalmente, uma filosofia de contornos espiritualistas, cujo objetivo central é a vivência do sagrado que conduz a união entre o Corpo-Mente-Alma e entre a alma e Deus.

O Yoga e a Meditação não são apenas técnicas fisiológicas e psíquicas que visam a saúde do corpo físico, mas fazem com que seus praticantes busquem um estado de identificação com a sua própria essência e ligação com o divino. Através de exercícios de posturas físicas, 
controle da respiração, controle da atenção e da mente, o praticante do yoga e da meditação realiza um processo introspectivo de mergulho dentro de si mesmo e encontro com sua natureza mais íntima e profunda. E esse mergulho é um encontro com a própria essência do sagrado, em torno do qual o ser se põe em contato não apenas consigo mesmo, mas com o próprio Ser eterno, soberano, perfeito, imutável e que as religiões chamam de Deus.

Se por um lado o Yoga e a Meditação podem ser vistos como um conjunto de técnicas terapêuticas e de condicionamento físico e mental visando a saúde e o bem estar, sendo utilizado como prática para o auxílio de pessoas com ansiedade, depressão e insônia, por outro lado não se pode perder de vista que o Yoga e a Meditação tem como fundamento uma face claramente religiosa, não de uma religião institucionalizada, mas no sentido de que oferece a busca pela libertação de maya (do mundo das ilusões) e pela manifestação da divindade interior, além de uma busca pela essência pessoal.

Nesse sentido as pesquisas sobre Yoga e Meditação tem muito a contribuir para os estudos das ciências da religião. A prática do Yoga e da Meditação une explicações científicas a partir das posturas físicas, práticas respiratórias, estados meditativos, com explicações religiosas que transcendem a compreensão do modelo biomédico de atenção à saúde e ultrapassa o reducionismo biológico, a cisão mente-corpo, que amputa o olhar sobre o binômio saúde/doença e a subjetividade do paciente. A junção do Yoga e da Meditação com as pesquisas em biomedicina ou na área das ciências psicofísicas promove uma ressignificação de tais práticas tanto no nível científico quanto religioso e, por isso, merece toda uma atenção especial no campo das ciências da religião.

\section{Referências Bibliográficas}

ADHIKARI, K. Study of Effect of Vipassana on Anxiety and Depression. International Journal of Psychology and Behavioral Science, 2(6), 274-276, 2012. Disponível em: <http://doi.org/10.5923/j.ijpbs.20120206.11>. Acesso em: 11 out. 2017.

ALTER, J. Yoga in Modern Indian: the Body between Science and Philosophy. New Jersey: Princeton University Press, 2004. 
BENSON, Herbert; KLIPPER, Miriam Z. The Relaxation Response. New York: HarperCollins Publishers Inc, 2000.

BOGELS, S.M.; SIJBERS, G.F.V.M.; VONCKEN, M. Mindfulness and task concentration training for social phobia: a pilot study. Journal of Cognitive Psychotherapy, n. 20, p. 33-44, 2006. Disponível em: <https://doi.org/10.1891/ jcop.20.1.33>. Acesso em: 13 ago. 2017

BROWN, K. W.; RYAN, R. M. The benefits of being present: Mindfulness and its role in psychological well-being. Journal of Personality and Social Psychology, vol. 84, n. 4, p. 822-848, 2003. Disponível em: <http://selfdeterminationtheory.org/SDT/documents/2003_BrownRyan.pdf>. Acesso em: 19 jun. 2017.

CARDOSO, R., [et. al.]. Meditation in health: An operational definition. Brain Research Protocols, vol. 14, n. 1, p. 58-60, 2004. Disponível em: <http:// dx.doi.org/10.1016/j.brainresprot.2004.09.002>. Acesso em: 05 mai. 2017.

CASEY, A.; BENSON, H. Mind your heart. New York: Free Press, 2004.

DAVIDSON, R. [et. al.]. Alterations in brain and immune function produced by mindfulness meditation. Laboratory for affective neuroscience. Psychosomatic Medicine, n. 65, p. 564-570, 2003. Disponível em: <https://www.ncbi. nlm.nih.gov/pubmed/12883106>. Acesso em: 13 ago. 2017

FEUERSTEIN, Georg. A tradição do Yoga: história, literatura, filosofia e prática. São Paulo: Pensamento, 2006.

GALVIN, J. A., [et. al.]. The relaxation response: Reducing stress and improving cognition in healthy aging adults. Complementary Therapies in Clinical Practice, vol. 12, n. 3, p. 186-191, 2006. Disponível em: <http://dx.doi. org/10.1016/j.ctcp.2006.02.004>. Acesso em: 19 jun. 2017.

GHAROTE, M. L. Técnicas de Yoga. Tradução de Danilo F. Santaella. Guarulhos: Phorte, 2000.

GOLEMAN, Daniel. A Mente Meditativa. 4. ed. São Paulo: Editora Ática, 1997.

HALL, Stuart. Identidade cultural na pós-modernidade. 11. ed. Rio de Janeiro: DP\&A, 2006.

KOZASA, E. A prática da meditação aplicada ao contexto da saúde. Saúde Coletiva, v.10 (3), 63-66, 2006. Disponível em: <www.redalyc.org/ pdf/842/84222224007.pdf>. Acesso em: 14 set. 2017.

, [et. al.]. Mind-body interventions for the treatment of insomnia: a review. Revista Brasileira de Psiquiatria, 32(4): 437-43, 2010. Disponível em: <http://dx.doi.org/10.1590/S1516-44462010000400018>. Acesso em: 14 set. 2017. 
do Yoga e da Meditação para a saúde do corpo e da alma

, [et. al.]. The effects of meditation-based interventions on the treatment of fibromyalgia. Current Pain and Headache Report, 16(5), 383-387, 2012. Disponível em: <https://www.ncbi.nlm.nih.gov/pubmed/22717699>. Acesso em: 14 set. 2017.

, [et. al.]. Meditation Training Increases brain efficiency in a attention task. Neuroimage, 59(1), 745-749, 2011. Disponível em: <https://www.ncbi. nlm.nih.gov/pubmed/21763432>. Acesso em: 14 set. 2017.

, [et. al.]. A Yoga and Compassion Meditation Program Reduces Stress in Familial Caregivers of Alzheimer's Disease Patients. Evidence-Based Complementary and Alternative Medicine, v.1, 35-43, 2013. Disponível em: <http:// dx.doi.org/10.1155/2013/513149>. Acesso em: 14 set. 2017.

JAIN, S. C.; [et. al.]. Effect of yoga training on exercise tolerance in adolescents with childhood asthma. Journal of Asthma, New Delhi, 28(6), p. 43742, 1991. Disponível em: <https://www.ncbi.nlm.nih.gov/pubmed/1744029>. Acesso em: 28 ago. 2017.

JAVNBAKHT, M.; HEJAZI, K.; GHASEMI, M. Effects of Yoga on depression and anxiety in women. Complement Ther Clin Pract, 15(2):102-4, 2009. Disponível em: < https://www.ncbi.nlm.nih.gov/pubmed/19341989>. Acesso em: 18 jul. 2017.

LEMOS, Kelly C. V. Meditação baseada em mindfulness e o método Feldenkrais ${ }^{\circledR}$ como terapias complementares no SUS: A Implementação da Política Nacional de Práticas Integrativas e Complementares no Instituto Nacional de Traumatologia e Ortopedia (INTO) Sob a Perspectiva dos Usuários. 2014. 131f. Dissertação (Mestrado em Psicossociologia). Programa de Pós-Graduação em Psicossociologia de Comunidades e Ecologia Social. Universidade Federal do Rio de Janeiro. Rio de Janeiro.

LIMA, Vânia C. L. O Yoga como caminho de elevação na espiritualidade e na saúde. 2010. 113f. Dissertação (Mestrado em Ciências das Religiões). Programa de Pós-Graduação em Ciências das Religiões. Universidade Federal da Paraíba. João Pessoa.

LUDERS, E., [et. al.]. The underlying anatomical correlates of long-term meditation: larger hippocampal and frontal volumes of gray matter. Neuroimage, v. 45, 672-678, 2009. Disponível em: <https://www.ncbi.nlm.nih.gov/ pubmed/19280691>. Acesso em: 11 jan. 2017.

MINISTÉRIO DA SAÚDE. Portaria n. 971, de 03 de maio de 2006. Aprova a Política Nacional de Práticas Integrativas e Complementares (PNPIC) no Sistema Único de Saúde. 2006. Disponível em: <http://bvsms.saude.gov.br/ bvs/saudelegis/gm/2006/prt0971_03_05_2006.html>. Acesso em 16/08/2017.

Portaria n. 849, de 27 de março de 2017. Inclui a Arteterapia, Ayurveda, Biodança, Dança Circular, Meditação, Musicoterapia, Naturopatia, Osteopatia, Quiropraxia, Reflexoterapia, Reiki, Shantala, Terapia Comunitária Integrati- 
va e Yoga à Política Nacional de Práticas Integrativas e Complementares. 2017. Disponível em: <http://www.lex.com.br/legis_27357131_PORTARIA_N_849 DE_27_DE_MARCO_DE_2017.aspx>. Acesso em 16/08/2017.

MORAES, Maria Regina C. Na fronteira entre religião e ciência: a meditação na grande imprensa brasileira. In: Simpósio Nacional da ABHR. Anais do XIV Simpósio Nacional da ABHR, vol. 14. Juiz de Fora, MG, 15 a 17 de abril de 2015.

PASHARD, O. Role of yoga in stress management. West Indian Med J,53(3):191-194, 2004. Disponível em: <https://www.ncbi.nlm.nih.gov/pubmed/15352751>. Acesso em: 18 jul. 2017.

PEÇANHA, Doris Leith; CAMPANA, Daniel Perdigão. Avaliação quali-quantitativa de intervenção com Yoga na promoção da qualidade de vida em uma universidade. Boletim Academia Paulista de Psicologia, vol. 78, n. 1, p. 199-218, 2010. Disponível em: <http://www.redalyc.org/articulo.oa?id=94615157014>. Acesso em: 08 fev. 2017.

RODRIGUES, M.R., [et. al.]. Estudos sobre o yoga. São Paulo: Phorte, 2006. SANTOS, Jair de Oliveira. Meditação: Fundamentos Científicos. Salvador: Faculdade Castro Alves, 2010.

SBISSA, Pedro Paulo M. Efeito da meditação mindfulness sobre a variabilidade da frequência cardíaca. 2010. 144f. Dissertação (Mestrado em Psicologia). Programa de Pós-Graduação em Psicologia. Universidade Federal de Santa Catarina. Florianópolis.

SIEGEL, Pamela. Yoga e Saúde: o desafio da introdução de uma prática não-convencional no SUS. 2010. 217f. Tese [Doutorado em Saúde Coletiva]. Faculdade de Ciências Médicas. Universidade Estadual de Campinas. Campinas.

SINGH, V.; [et. al.]. Effect of yoga breathing exercises (pranayama) on airway reactivity in subjects with asthma. Lancet, 335, p. 1381-83, jun. 1990. Disponível em: <https://www.ncbi.nlm.nih.gov/pubmed/1971670>. Acesso em: 28 ago. 2017.

SIMÕES, Roberto Serafim. Fisiologia da Religião: uma análise sobre vários estudos da prática religiosa do Yoga. Dissertação (Mestrado em Ciências da Religião). Pontifícia Universidade Católica de São Paulo. São Paulo, 2011.

TEOTIA, Harendra S.; TEOTIA, Rita. Yoga e Saúde. Dieta, relaxamento, respiração, posturas, meditação \& estresse. Campina Grande-PB: Oriental Yoga Center, 2000.

TONIOL, Rodrigo. Espiritualidade que faz bem. Pesquisas, políticas públicas e práticas clínicas pela promoção da espiritualidade como saúde. Sociedad y Religión, Buenos Aires, n.43, vol XXV, pp. 110-143, mayo 2015. Disponível em: <http://www.scielo.org.ar/scielo.php?pid=S1853-70812015000100005\&script=sci_arttext $>$. Acesso em: 05 mai. 2017. 
VORKAPIC, Camila F.; RANGÉ, Bernard. Os benefícios do yoga nos transtornos de ansiedade. Revista Brasileira de Terapias Cognitivas, vol. 7, n. 1, p. 50-54, jun. 2011. Disponível em: <http://pepsic.bvsalud.org/scielo. php?pid=S1808-56872011000100009\&script=sci_arttext $>$. Acesso em: 07 jun. 2017.

WILLIAMS, J. M; SWALES, M. The use of mindfulness-based approaches for suicidal patients. Archives of Suicide Research; v. 8, n. 4, p. 315-329, 2004. Disponível em: <http://dx.doi.org/10.1080/13811110490476671>. Acesso em: 13 ago. 2017 